\title{
Investigation of the corrosion behavior of carbon steel with a protective superhydrophobic coating by impedance spectroscopy method
}

\author{
L.E. Tsygankova, ${ }^{1}$ (i) $*$ M.N. Uryadnikova, ${ }^{1}$ V.I. Kichigin $^{2}$ \\ and L.D. Rodionova ${ }^{1}$
}

${ }^{1}$ Derzhavin State University, Internatsyonalnaya str., 33, 392000 Tambov, Russian Federation

${ }^{2}$ Perm State University, str. Bukireva, 15, 614990 Perm, Russian Federation

*E-mail: vits21@mail.ru

\begin{abstract}
The impedance spectra of a steel electrode with a superhydrophobic coating obtained at the corrosion potential $E_{\text {cor }}$ in solutions of $\mathrm{NaCl}(50 \mathrm{~g} / \mathrm{L})$ and NACE $\left(0.25 \mathrm{~g} / \mathrm{L} \mathrm{CH}_{3} \mathrm{COOH}, 5 \mathrm{~g} / \mathrm{L}\right.$ $\mathrm{NaCl}, \mathrm{pH}=3.6)$ in the absence and presence of $400 \mathrm{mg} / \mathrm{L} \mathrm{H}_{2} \mathrm{~S}$ are analyzed. Frequency impedance spectra were recorded after 15 minutes of exposure in the working solution and every day for 168 hours without removing electrode from the solution. Three equivalent circuits are considered that model the processes occurring on the electrode at the corrosion potential. The most acceptable circuit is one that is characterized by smaller deviations of the calculated impedance values from the experimental ones. It is a parallel connection of four circuits: electrical double layer capacitance $C_{\mathrm{dl}}$; a series combination of resistance $R_{\mathrm{c}}$ and diffusion impedance $Z_{\mathrm{d}}$ simulating the cathodic process; anodic reaction resistance $R_{\mathrm{a}}$; a series combination of $R_{1}$ and $C_{1}$ (the elements $R_{\mathrm{a}}, R_{1}, C_{1}$ model the anodic process). This equivalent circuit can describe impedance spectra with three time constants, which are observed in some cases experimentally. Calculation of corrosion rates in the studied media using impedance data showed that in the NACE environment in the presence of $\mathrm{H}_{2} \mathrm{~S}$ after 24 hours of exposure, the corrosion rate is $40 \%$ lower, and after 168 hours, 2.3 times higher than in the absence of $\mathrm{H}_{2} \mathrm{~S}$. These results are in qualitative agreement with those obtained by the method of polarization curves. The observed changes in the parameters of the equivalent circuit with time are mainly associated with the influence of two processes - gradual degradation of the hydrophobic coating and the growth of a protective film of corrosion products.
\end{abstract}

Keywords: steel, superhydrophobic coating, corrosion rate, solution, hydrogen sulfide, impedance spectroscopy, equivalent circuit.

Received: January 16, 2020. Published: February 10, 2021

doi: $\underline{10.17675 / 2305-6894-2021-10-1-11}$

\section{Introduction}

Over the past two decades, intensive research is carried out on the protection of metals against corrosion by means of hydrophobic and superhydrophobic coatings (SHPC). It is 
known that for the formation of superhydrophobic coatings on metals, it is necessary to create a multimodal surface roughness, followed by the application of materials with low surface energy. The latter are organic silanes, fluorocarbons, fatty acids or fatty amines with a long hydrocarbon chain. Various methods are used to create surface roughness, such as chemical etching [1], chemical vapor deposition [2], nanosecond laser surface treatment [3], template methods [4], etc. Multilevel roughness contributes to the trapping of air bubbles, which prevent the access of the liquid medium to the metal surface and cause a high contact angle $\theta_{c}\left(\theta_{c}>150^{\circ}\right)$. Nevertheless, hydrophobic and superhydrophobic coatings do not completely exclude metal contact with the electrolyte solution. It is believed that they have pores, or the so-called areas of the wetted surface, the total share of which can reach $10 \%$ $[5,6]$.

In most cases, when assessing the protective ability of hydrophobic and superhydrophobic films, many authors, as a rule, neglect the data of direct corrosion tests. Evaluation is usually done by measuring the polarization curves and determining the corrosion rate $i_{\text {cor }}$ by extrapolating their Tafel sections to the corrosion potential. Or the method of impedance spectroscopy is used and $i_{\text {cor }}$ is calculated using the value of the polarization resistance $R_{\mathrm{p}}$ in accordance with the Stern-Geary equation:

$$
i_{\text {cor }}=\frac{b_{\mathrm{a}} b_{\mathrm{c}}}{2.303\left(b_{\mathrm{a}}+b_{\mathrm{c}}\right)} \cdot \frac{1}{R_{\mathrm{p}}}
$$

where $b_{\mathrm{a}}$ and $b_{\mathrm{c}}$ are the Tafel slope coefficients of the anodic and cathodic branches of the polarization curves. However, as can be seen, in this case the values of the slope coefficients of the Tafel sections of the polarization curves are also necessary.

The protective efficacy of SHPC on metals is most often studied in neutral chloride media. The method of polarization curves was used to study the corrosion rate of carbon steel in the presence of superhydrophobic coatings based on fluoroxysilane in solutions of $\mathrm{NaCl}(50 \mathrm{~g} / \mathrm{L})$ [7] and NACE [8] in the absence and presence of $400 \mathrm{mg} / \mathrm{L} \mathrm{H}_{2} \mathrm{~S}$ in both cases. In the NACE environment, during 96 hours of electrode exposure in the presence of $\mathrm{H}_{2} \mathrm{~S}$, the corrosion rate of steel was $25 \%$ lower than in the absence of $\mathrm{H}_{2} \mathrm{~S}$, after 120 hours it was already $25 \%$ higher and continued to increase over time. In $\mathrm{NaCl}$ solution for 48 hours in the presence of $\mathrm{H}_{2} \mathrm{~S}$, the corrosion rate of steel was 15-20\% higher than in the absence of $\mathrm{H}_{2} \mathrm{~S}$, after 72 and 96 hours the corrosion rate values were practically the same. Corrosion potentials shifted to the region of more negative values over time. The observed features are explained by the formation of a protective sulfide film of corrosion products on the surface and in wetted areas.

The aim of this work is to study the corrosive behavior of carbon steel in the presence of a superhydrophobic coating in the above media using impedance spectroscopy. 


\section{Experimental}

The corrosion behavior of St3 carbon steel (chemical composition, mass \%: C $-0.20 ; \mathrm{Mn}-$ $0.50 ; \mathrm{Si}-0.15 ; \mathrm{P}-0.04 ; \mathrm{S}-0.05 ; \mathrm{Cr}-0.30 ; \mathrm{Ni}-0.20 ; \mathrm{Cu}-0.20 ; \mathrm{Fe}-98.36)$ with a superhydrophobic coating was studied in $\mathrm{NaCl}(50 \mathrm{~g} / \mathrm{L})$ and NACE $\left(0.25 \mathrm{~g} / \mathrm{L} \mathrm{CH}_{3} \mathrm{COOH}\right.$, $5 \mathrm{~g} / \mathrm{L} \mathrm{NaCl}, \mathrm{pH}=3.6$ ) solutions in the absence and presence of $400 \mathrm{mg} / \mathrm{L} \mathrm{H}_{2} \mathrm{~S}$. The superhydrophobic coating was applied in the Laboratory of Surface Forces at the Institute of Physical Chemistry and Electrochemistry, Russian Academy of Sciences, to samples pressed into a fluoroplastic frame with a working surface area close to $0.5 \mathrm{~cm}^{2}$. To obtain a coating, the working surface was textured with a nanosecond IR laser with the following laser processing parameters: pulse duration $50 \mathrm{~ns}$, pulse frequency $20 \mathrm{kHz}$, peak pulse power $0.95 \mathrm{~mJ}$, linear laser beam rate $50 \mathrm{~mm} / \mathrm{s}$, scanning density 150 lines per $\mathrm{mm}$. Immediately after texturing with the indicated parameters, the surface showed super hydrophilic properties - a drop of water when it touched such a surface instantly spread into a thin film. To impart superhydrophobic properties to the surface, the surface energy of the formed texture was lowered by chemisorption of the hydrophobizing agent $\mathrm{CF}_{3}\left(\mathrm{CF}_{2}\right)_{6}\left(\mathrm{CH}_{2}\right) \mathrm{O}\left(\mathrm{CH}_{2}\right)_{2} \mathrm{C}\left(\mathrm{OCH}_{3}\right)_{3}$ from the vapor phase, followed by drying of the resulting coating at $130^{\circ} \mathrm{C}$ for 1 hour. The wetting angle on the obtained superhydrophobic samples was $165 \pm 2^{\circ}$, and the rolling angle for droplets with a volume of $15 \mu \mathrm{L}$ was $3 \pm 1^{\circ}$. The samples under study (due to design features) were not subjected to ultraviolet irradiation in the presence of ozone plasma for strengthening chemisorption of a hydrophobic agent [9].

Impedance spectra were studied in the frequency range of $10 \mathrm{kHz}-0.01 \mathrm{~Hz}$ with an alternating voltage amplitude of $10 \mathrm{mV}$, using an electrochemical measuring complex from Solartron (UK) consisting of a 1255 SI impedance analyzer and a SI 1287 potentiostat. Frequency impedance spectra were recorded after 15 minutes of electrode exposure in the working solution (the time required to establish the corrosion potential $E_{\text {cor }}$ ) and every day for 168 hours without removing from the solution.

The processing of the impedance measurement results was carried out using the ZView 3.0 program, which makes it possible to carry out calculations according to any equivalent circuits with the number of elements up to 20 . To avoid the situation of finding a local minimum of the function, the calculation was repeated several times with different initial values of the circuit elements. The criterion for evaluating the circuits is the standard deviation s; the equivalent circuit is considered satisfactory at $s \leq 5 \%$.

\section{Results}

\subsection{NACE solution}

The experimental spectra of the impedance of a steel electrode with a superhydrophobic coating, obtained at the corrosion potential $E_{\text {cor }}$, are shown in Figure 1. It can be seen that with an increase in the exposure time $t$ of the electrode in a corrosive medium, the impedance gradually increases at $t \geq 24 \mathrm{~h}$, which indicates a decrease in the corrosion rate with time. At high frequencies, as follows from the Bode plots (Figure 1,b), there is a small capacitive arc, 
which can be associated with SHPC or with the separation of the time constants of the cathodic and anodic processes.

At $t=15 \mathrm{~min}$, an anomalous impedance spectrum is observed: up to a frequency of $f \approx 0.1 \mathrm{~Hz}$, the impedance increases, and with a further decrease in frequency, significant deviations from the capacitive arc begin. At a frequency of $0.05 \mathrm{~Hz}$, the impedance reaches the value $|Z| \approx 550 \mathrm{Ohm} \cdot \mathrm{cm}^{2}$ (real component $\sim 500 \mathrm{Ohm} \cdot \mathrm{cm}^{2}$ ), and then begins to decrease rapidly and at a frequency of $0.01 \mathrm{~Hz}$, the impedance modulus is only $250 \mathrm{Ohm} \cdot \mathrm{cm}^{2}$. At the same time, after a day, it rises to $\sim 600 \mathrm{Ohm} \cdot \mathrm{cm}^{2}$ (at low frequencies). A similar impedance spectrum was observed, for example, by the authors of [10] for a corroding copper electrode in a solution with $\mathrm{pH}=1$, containing $0.5 \mathrm{M} \mathrm{Cl}^{-}$, at a short exposure time; this was attributed to a decrease in the polarization resistance with time due to the dissolution of the native oxide film on the electrode.
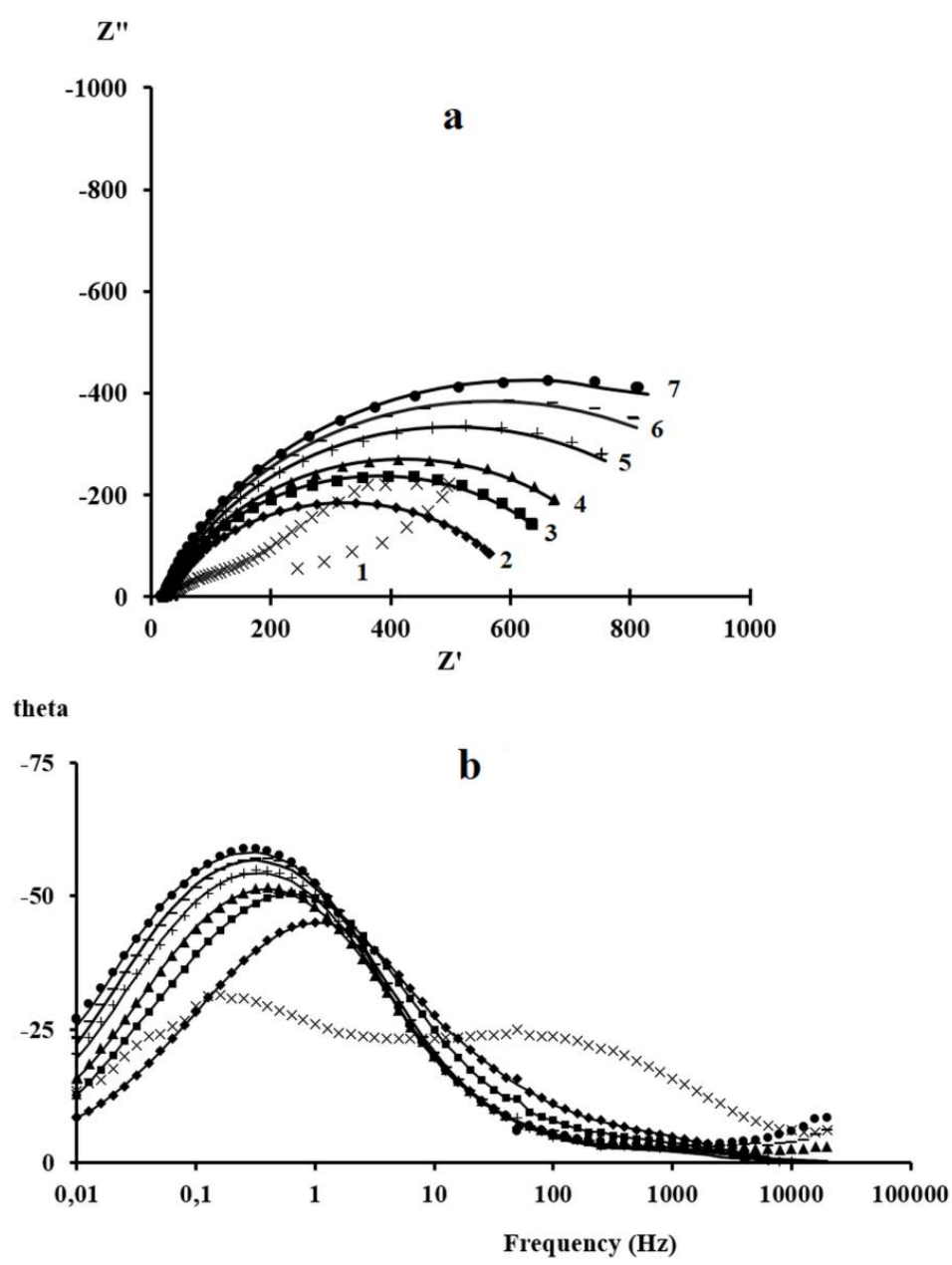

Figure 1. Nyquist (a) and (b) Bode diagrams of a steel electrode with a superhydrophobic coating in NACE environment at $E_{\text {cor. }}$ The exposure time of the electrode in the solution, h: $1-0.25 ; 2-24 ; 3-48 ; 4-72 ; 5-96 ; 6-120 ; 7-168$. Points correspond to experimental data, solid lines - data calculated on the basis of the equivalent circuit C. $Z^{\prime}, Z^{\prime \prime}, \Omega \cdot \mathrm{cm}^{2}$; theta, degrees. 
It can be assumed that the anomaly at $t=15 \mathrm{~min}$ is associated with the nonstationarity of the system under study: in the first minutes after immersion of the electrode in the solution, the state of the coating and the electrode surface undergoes, probably, the most rapid changes, therefore, during the measurement of the impedance spectrum, a rather rapid drift of electrochemical characteristics occurs, which leads to significant deviations of the points at the lowest frequencies.

The appearance of the impedance graph at $t=15 \mathrm{~min}$ does not necessarily mean that during the measurement of the impedance spectrum, a rapid increase in the corrosion rate occurs due to degradation of the coating (this does not correspond to the increase in impedance at $t \geq 24 \mathrm{~h}$ ). Significant impedance reduction at low frequencies can occur when the impedance is measured at corrosion potential in potentiostatic mode using a Solartron Frequency Response Analyzer, and the electrode potentials then maintained equal to $E_{\text {cor }}$ just prior to the impedance spectrum measurement. If during the measurement of the spectrum $E_{\text {cor }}$ shifts in the negative direction (namely, such a shift in $E_{\text {cor }}$ was recorded experimentally at short exposure times), then at the end of the measurement of the frequency characteristic of the impedance, the electrode becomes anodically polarized; the anodic current density is higher than the corrosion current density, which explains the observed decrease in impedance at low frequencies.

The validity of experimental impedance data can be checked with the Kramers-Kronig relations [11]. In practice, it is possible to use the measurement model method [12]. If the experimental impedance spectrum can be described using some electric circuit that corresponds to the Kramers-Kronig relations, then the experimental data are also consistent with these relations. The Voigt circuit can be used as a measurement model (Figure 2).

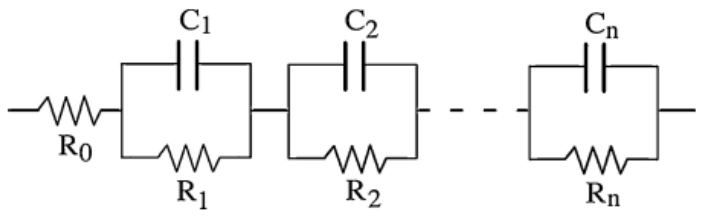

Figure 2. Voigt's circuit.

The impedance hodograph was processed for NACE solution at an exposure time of 15 min using the Voigt circuit with the number of RC elements from $n=3$ to $n=6$. To achieve a sufficiently good fit, in the Voigt circuit, instead of the capacitors $C_{i}$, we used a constant phase element CPE, whose admittance $Y_{\mathrm{CPE}}$ is [13]

$$
Y=Q(j \omega)^{p}
$$

where $Q$ is a frequency-independent factor, $p$ is an exponent $(p<1), \omega$ is the angular frequency of an alternating current, $\omega=2 \pi f$. The result of using the Voigt model is shown in Figure 3. Although there is formal agreement between the model and the experimental impedance spectrum, some results indicate that the Voigt model is in fact not applicable. These are the following results: a) negative time constant for one of the RQ-elements $(R<0$, 
$Q>0$ ), although the entire hodograph is in the capacitive half-plane, in one quadrant; negative time constant is obtained not only for $n=5$ (Figure 3), but also for other $n$, as well as for the Voigt circuit of RC elements; b) the parameter $p$ for one of the CPE has a value greater than 2, which has no physical meaning, since this parameter must be between -1 and 1 .

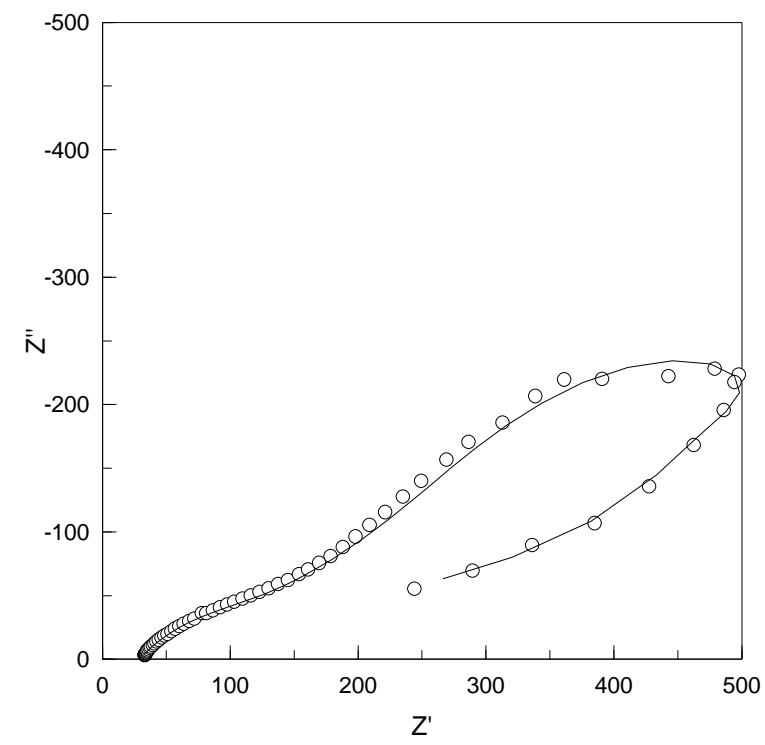

Figure 3. Approximation of the impedance spectrum in NACE solution at $t=15 \mathrm{~min}$ using the Voigt circuit of five RQ elements. The impedance is measured to a frequency of $0.01 \mathrm{~Hz}$. $Z^{\prime}$, $Z^{\prime \prime}, \Omega \cdot \mathrm{cm}^{2}$.

Since the Voigt circuit is essentially not suitable for describing the full impedance spectrum at a short exposure time, the full impedance hodograph, including points at low frequencies, is not Kramers-Kronig transformable; therefore, this shape of the hodograph does not reflect the actual electrochemical behavior, most likely due to the unsteady state of the system. However, at $t=15 \mathrm{~min}$, we can process the hodograph without low-frequency points. Such a reduced impedance spectrum (down to $f \approx 0.2 \mathrm{~Hz}$ ) approaches the spectrum corresponding to an actual exposure time of $15 \mathrm{~min}$, non-stationary effects for it are minimal, and when it is processed using the Voigt model, negative time constants or $p>1$ are not observed.

\subsection{NACE solution $+400 \mathrm{mg} / \mathrm{L} \mathrm{H}_{2} \mathrm{~S}$}

The experimental spectra of the impedance of a steel electrode with SHPC, obtained in a hydrogen sulfide-containing NACE solution at $E_{\text {cor }}$, are shown in Figure 4 . On the Nyquist plot at $t=15 \mathrm{~min}$, the same anomaly is observed as in a solution without $\mathrm{H}_{2} \mathrm{~S}$, and everything that was said earlier about the shape of the impedance plot at a short exposure time is also true here. 

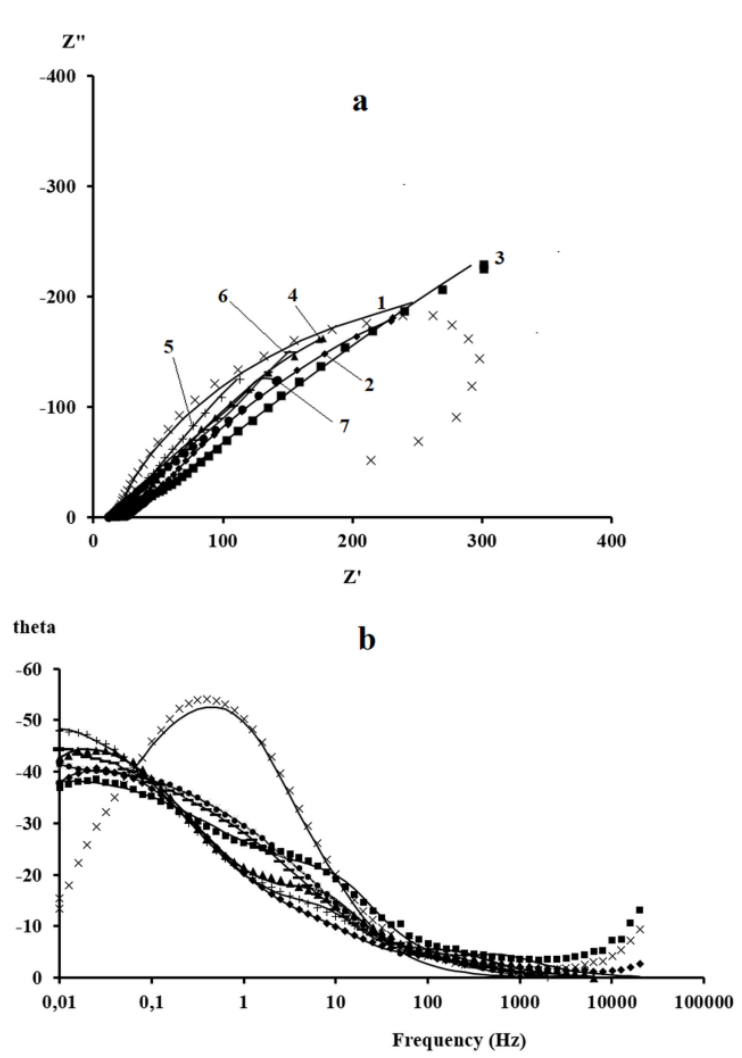

Figure 4. Nyquist (a) and Bode (b) diagrams of a steel electrode with a superhydrophobic

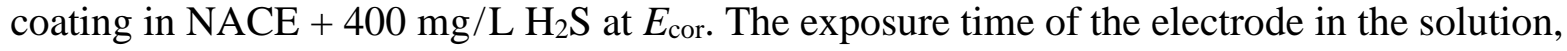
h: $1-0.25 ; 2-24 ; 3-48 ; 4-72 ; 5-96 ; 6-120 ; 7-168$. Points correspond to experimental data, solid line - data calculated on the basis of the equivalent circuit C. $Z^{\prime}, Z^{\prime \prime}, \Omega \cdot \mathrm{cm}^{2}$; theta, degrees.

With increasing exposure time, a non-monotonic change in the size of the capacitive arc is observed. However, these arcs are less than a quarter of a circle, and extrapolation to $f \rightarrow 0$ (by approximation by the equation of a circle) to estimate the polarization resistance $R_{\mathrm{p}}$ and corrosion rate can give significant error. More reasonable estimates of $R_{\mathrm{p}}$ are possible using an adequate equivalent circuit (see below). As follows from the Bode plots (Figure 4,b), up to three time constants are observed, which characterize the impedance spectra in a NACE $+400 \mathrm{mg} / \mathrm{L} \mathrm{H}_{2} \mathrm{~S}$ solution.

\section{3. $\mathrm{NaCl}$ solution}

At $t=15 \mathrm{~min}$, the same impedance spectrum anomaly is observed as in the NACE solution, but the decrease in impedance at low frequencies is less pronounced (Figure 5,a). With increasing exposure time, there is a tendency for the size of the capacitive arc to increase. The impedance spectra are characterized by two time constants (Figure 5,b). 

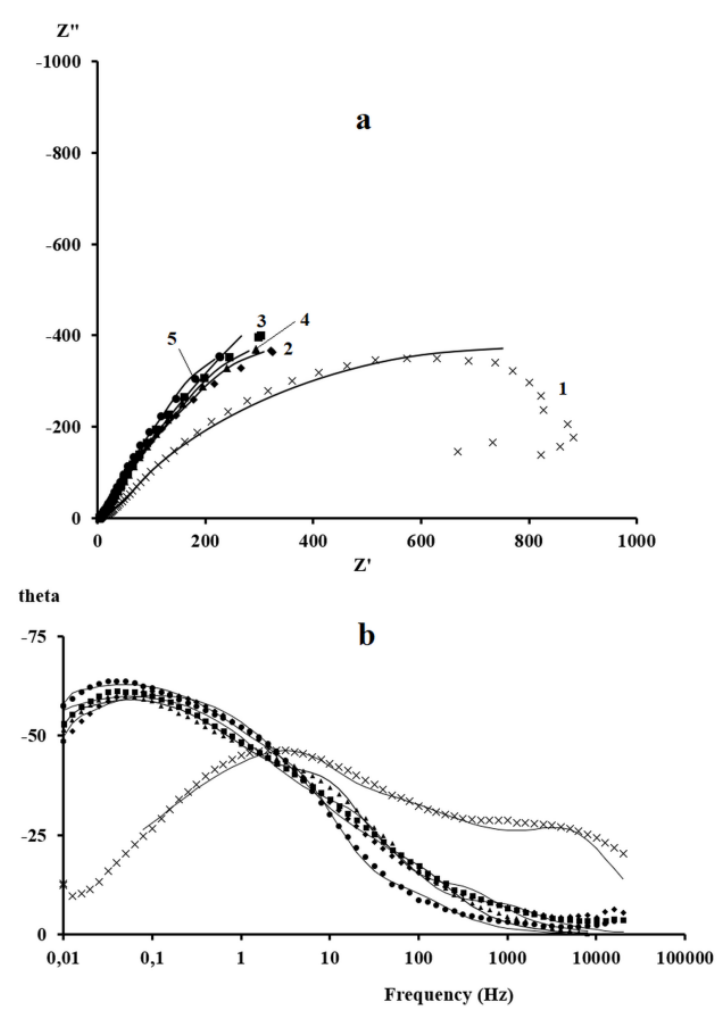

Figure 5. Nyquist (a) and Bode (b) diagrams of a steel electrode with a superhydrophobic coating in $\mathrm{NaCl}(50 \mathrm{~g} / \mathrm{L})$ solution at $E_{\text {cor. }}$. The exposure time of the electrode in the solution, h: $1-0.25 ; 2-24 ; 3-48 ; 4-72 ; 5-168$. Points correspond to experimental data, solid line data calculated on the basis of the equivalent circuit C. $Z^{\prime}, Z^{\prime \prime}, \Omega \cdot \mathrm{cm}^{2}$; theta, degrees.

\section{4. $\mathrm{NaCl}$ solution $+400 \mathrm{mg} / \mathrm{L} \mathrm{H}_{2} \mathrm{~S}$}
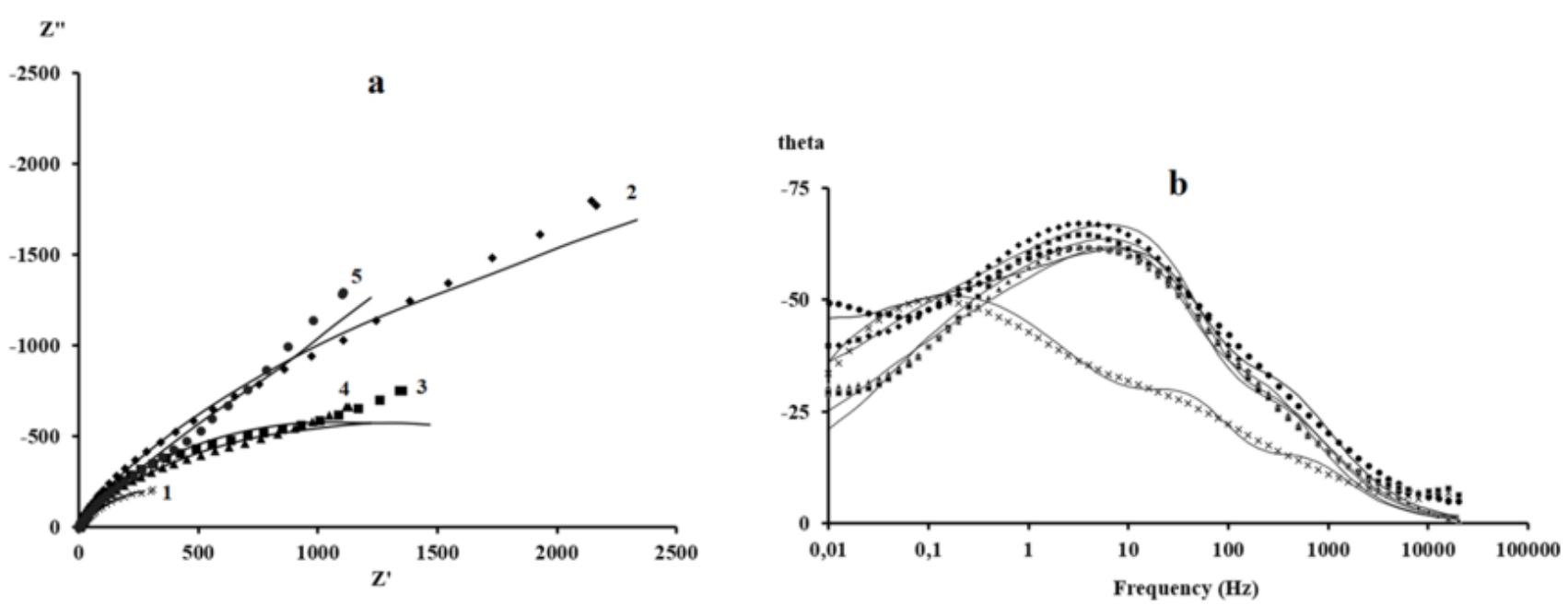

Figure 6. Nyquist (a) and Bode (b) diagrams of a steel electrode with a superhydrophobic coating in $\mathrm{NaCl}+400 \mathrm{mg} / \mathrm{L}$ solution at $E_{\text {cor. }}$ The exposure time of the electrode in the solution, h: $1-0.25 ; 2-24 ; 3-48 ; 4-72 ; 5-168$. Points correspond to experimental data, solid line - data calculated on the basis of the equivalent circuit $C$. $Z^{\prime}, Z^{\prime \prime}, \Omega \cdot \mathrm{cm}^{2}$; theta, degrees. 
In this solution, the impedance values are relatively large (Figure 6,a). There was no anomaly in the impedance plot at $t=15 \mathrm{~min}$.

\section{Selection of the equivalent electrical circuit}

Consider the equivalent electrical circuits of two types, the general view of which is shown in Figure 7. The equivalent circuit in Figure 7a is a circuit that simulates an electrode, on the surface of which there is a dielectric coating containing ionic conduction paths (for example, pores). Here $C_{\mathrm{f}}$ is the coating capacitance equal to $\varepsilon_{0} \varepsilon / d$ ( $\varepsilon$ is the dielectric constant of the coating material, $\varepsilon_{0}$ is the electrical constant, $d$ is the coating thickness); $R_{\mathrm{f}}$ is the film resistance (resistance of solution in the film pores); $Z_{1}$ is the impedance of electrochemical processes at the metal/solution interface, i.e. processes at the bottom of the pores in the coating. When measuring the impedance at the corrosion potential, the impedance $Z_{1}$ should contain parallel connected impedances of the anodic $Z_{\mathrm{a}}$ and cathodic $Z_{\mathrm{c}}$ processes, as well as the impedance of the electric double layer $[14,15]$. The type of $Z_{\mathrm{a}}$ and $Z_{\mathrm{c}}$ in $Z_{1}$ is determined by the mechanisms of anodic and cathodic partial reactions.
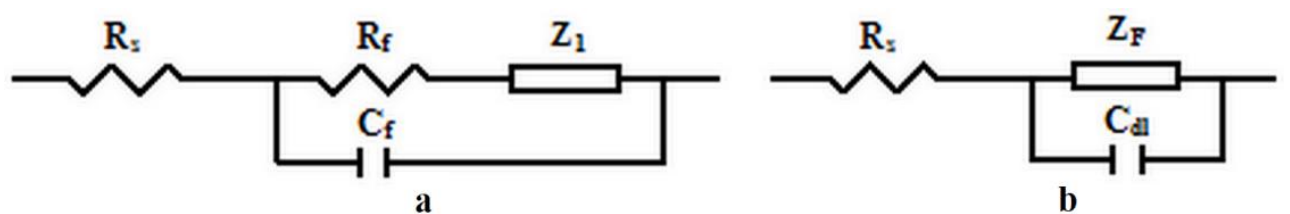

Figure 7. Two types of equivalent circuits.

The second type of equivalent circuits (Figure 7,b) is a conventional circuit containing, in addition to the solution resistance $R_{\mathrm{s}}$, the Faraday impedance $Z_{\mathrm{F}}$ and the capacitance of the double layer $C_{\mathrm{dl}}$. With $E_{\mathrm{cor}}$, the Faraday impedance is also a parallel connection of $Z_{\mathrm{a}}$ and $Z_{\mathrm{c}}$. In both types of equivalent circuits, CPE can be used instead of $C_{\mathrm{dl}}$ or $C_{\mathrm{f}}$. There are some reasons for using circuits like Figure 7,b: superhydrophobic layers are very thin, and they can be considered as a very thin layer between the metal and the nearest layer of electrolyte molecules and ions, modifying the structure of the electric double layer, but not changing the nature of the processes at the interface; defects in a thin coating are distributed fairly evenly, and the distribution of electrode processes over the electrode surface is also fairly uniform; according to one of the SHPC models, up to $10 \%$ of the hydrophobized surface can be occupied by open hydrophilic areas $[5,6,16]$. In this case, the superhydrophobic coating manifests itself through its influence on the kinetic characteristics of electrode reactions. Let us note some systems similar to SHPC, for which equivalent circuits of the type in Figure 7,b were used. One of such systems is self-assembled monolayers (SAM). These layers, although they may contain defects, are dense, highly ordered, and act as a hydrophobic barrier layer that prevents the metal from contacting with corrosive electrolyte ions [17, 18]. Despite this, in a number of works [19-21], for electrodes on the surface of which SAMs were formed, the same simple equivalent circuits (of the type in Figure 7,b) were used as for substrates without SAM films. The possibility of using equivalent circuits such as Figure 7,b 
in the case of the formation of polymolecular adsorption layers of corrosion inhibitors, when the capacity of the double layer takes values less than $1 \mu \mathrm{F} / \mathrm{cm}^{2}$, was also shown [22].

Various equivalent circuits for electrodes with superhydrophobic coatings were given in the literature [23-27], but circuit A (Figure 8) was used more often. It can be attributed to the first type (Figure 7a). Circuit A assumes that both the anodic and cathodic processes on the corroding electrode proceed with kinetic control. In the case of the SHPC under study, circuit A with two CPEs (instead of $C_{\mathrm{f}}$ and $C_{\mathrm{dl}}$ ) describes the experimental spectra well, but the parameter $p$ in the CPE used instead of $C_{\mathrm{f}}$ turns out to be very low $(0.66-0.53)$, which does not correspond to the capacitive type of CPE. In addition, in some cases, three time constants appear on the experimental spectra (for example, NACE $+\mathrm{H}_{2} \mathrm{~S}$; Figure 4), and circuit A cannot describe such cases. For these reasons, we consider this equivalent circuit unsuitable for describing the impedance data for the studied SHPC.

In this regard, another equivalent circuit of the first type was tested - circuit B (Figure 8). In this circuit, the impedance $Z_{1}$ is a circuit from [28], applicable to a corrosion system in a neutral medium, in which oxygen reduction is the cathodic process. The cathodic process is modeled by an Ershler-Randles circuit of series-connected resistance $R_{\mathrm{c}}$ and diffusion impedance $Z_{\mathrm{d}}$. The anodic process is simulated in a simplified manner by the ohmic resistance $R_{\mathrm{a}}$. The use of the ohmic resistance $R_{\mathrm{a}}$ as the Faraday impedance of the anodic reaction does not negate its multistep nature, but imposes a number of assumptions regarding the rate-determining step, the preceding and subsequent steps [29, 30].

Table 1. Values of the parameters of the equivalent circuit B for a steel electrode with SHPC in NACE solution.

\begin{tabular}{|c|c|c|c|c|c|c|}
\hline \multirow{2}{*}{ Parameter } & \multicolumn{6}{|c|}{ Exposure time, $\mathbf{h}$} \\
\hline & 24 & 48 & 72 & 96 & 120 & 168 \\
\hline$C_{\mathrm{f}}, \mu \mathrm{F} / \mathrm{cm}^{2}$ & 58.4 & 80 & 83.8 & 118,6 & 49.5 & 35.6 \\
\hline$R_{\mathrm{f}}, \mathrm{Ohm} \cdot \mathrm{cm}^{2}$ & 5.2 & 3.2 & 2.3 & 2.3 & 1.9 & 1.7 \\
\hline$C_{\mathrm{dl}}, \mu \mathrm{F} / \mathrm{cm}^{2}$ & 183 & 350 & 527 & 709 & 563 & 516 \\
\hline$R_{\mathrm{a}}, \mathrm{Ohm} \cdot \mathrm{cm}^{2}$ & 594 & 731 & 810 & 992 & 1110 & 1200 \\
\hline$R_{\mathrm{c}}, \mathrm{Ohm} \cdot \mathrm{cm}^{2}$ & 8.4 & 4.4 & 4.6 & 5.0 & 2.8 & 2.7 \\
\hline$R_{\mathrm{d}}, \mathrm{Ohm} \cdot \mathrm{cm}^{2}$ & 12030 & 12200 & 12010 & 14780 & 13430 & 13210 \\
\hline$\tau_{\mathrm{d}}, \mathrm{s}$ & 95 & 142 & 155 & 214 & 178 & 174 \\
\hline$p_{\mathrm{d}}$ & 0.69 & 0.71 & 0.72 & 0.72 & 0.75 & 0.76 \\
\hline$s, \%$ & 1.5 & 3.1 & 2.0 & 0.8 & 0.8 & 3.8 \\
\hline
\end{tabular}

Some results of processing the impedance spectra using circuit B are shown in Table 1. In Table 1: $C_{\mathrm{dl}}$ is the capacitance of the double layer in open areas of the electrode, $R_{\mathrm{a}}$ is the 
resistance of the anodic reaction, $R_{\mathrm{c}}$ is the resistance of the cathodic reaction, $R_{\mathrm{d}}, \tau_{\mathrm{d}}$ and $p_{\mathrm{d}}$ are the parameters of the diffusion impedance

$$
Z_{\mathrm{d}}=R_{\mathrm{d}} \frac{\tanh \left(j \omega \tau_{\mathrm{d}}\right)^{p_{\mathrm{d}}}}{\left(j \omega \tau_{\mathrm{d}}\right)^{p_{\mathrm{d}}}}
$$

Low values of $R_{\mathrm{f}}$ can be noted, which is in qualitative agreement with the very shallow depth of possible pores. However, if, when evaluating the total pore cross section $S_{\mathrm{p}}$ in the coating on an electrode with an area of $1 \mathrm{~cm}^{2}$, we take even the smallest value of $R_{\mathrm{f}}$ obtained for circuit B $\left(1.7 \mathrm{Ohm} \cdot \mathrm{cm}^{2}\right.$ at large $\left.t\right)$, then we obtain $S_{\mathrm{p}}=\rho d / R_{\mathrm{f}} \approx 6 \cdot 10^{-6}$, where $\rho$ is the resistivity of the solution (for NACE $\rho \approx 100 \mathrm{Ohm} \cdot \mathrm{cm}$ ), $d$ is the pore length (the thickness of the coating is taken equal to $1 \mathrm{~nm}$ ). Thus, an estimate of the porosity of the coating gives a value of $0.0006 \%$, which appears to be too small. An estimate of the capacitance $C_{\mathrm{f}}$ using the above formula at $\varepsilon=3-4$ and a coating thickness of $1 \mathrm{~nm}$ gives $C_{\mathrm{f}}=2.6-3.5 \mu \mathrm{F} / \mathrm{cm}^{2}$ (when the surface is completely filled with the hydrophobic coating itself), while, for example, at $t=24 \mathrm{~h} C_{\mathrm{f}}=58 \mu \mathrm{F} / \mathrm{cm}^{2}$ (Table 1), that is, the $C_{\mathrm{f}}$ values obtained for circuit B seem to be high. Also the values of the $C_{\mathrm{dl}}$ capacitance in circuit B are high - several hundred $\mu \mathrm{F} / \mathrm{cm}^{2}$ (and in a NACE $+\mathrm{H}_{2} \mathrm{~S}$ solution - up to $\sim 2000 \mu \mathrm{F} / \mathrm{cm}^{2}$ ), while in accordance with the physical meaning of the $C_{\mathrm{dl}}$ capacitance in circuit B it should be determined by the relation $C_{\mathrm{dl}}=\mathrm{C}_{\mathrm{dl}, 0}(1-\theta)$, where $C_{\mathrm{dl}, 0}$ is the specific capacity of the double layer on an uncoated electrode under the same conditions, $\theta$ is the fraction of the surface occupied by the hydrophobic film. At $\theta>90 \%$ and $C_{\mathrm{dl}, 0}$ equal to several tens of $\mu \mathrm{F} / \mathrm{cm}^{2}$ (typical values of double-layer capacitance for solid electrodes), one should expect $C_{\mathrm{dl}}$ values ranging from several units of $\mu \mathrm{F} / \mathrm{cm}^{2}$ to several tenths of $\mu \mathrm{F} / \mathrm{cm}^{2}$, which is much less than $C_{\mathrm{dl}}$ in Table 1 . The same applies to the elements of the Faraday impedance in circuit B: the smaller the total cross-section of the pores, the more resistances and less capacitance should be.

In connection with the indicated difficulties in matching the observed values of $R_{\mathrm{f}}, C_{\mathrm{f}}$, $C_{\mathrm{dl}}$ with their physical meaning, let us turn to the equivalent circuit of the second type circuit $\mathrm{C}$ (Figure 8). This circuit was proposed in [31] to describe the impedance of corroding electrodes in neutral media. It has a common basis with the circuit from [28], but differs in the presence of elements $R_{1}$ and $C_{1}$, which refer to the Faraday impedance of the anodic partial reaction; the elements $R_{\mathrm{a}}, R_{1}$ and $C_{1}$ more accurately describe the impedance response of a multistep iron ionization reaction compared to the resistance $\mathrm{R}_{\mathrm{a}}$ alone. The diffusion impedance in circuit $\mathrm{C}$ is also described by relation (3), the capacitance $C_{\mathrm{dl}}$ in circuit $\mathrm{C}$ can be represented as

$$
C_{\mathrm{dl}}=C_{\mathrm{dl}, 0}(1-\theta)+C_{\mathrm{ld}, 1} \theta
$$

where $C_{\mathrm{dl}, 1}$ is the capacitance of the electric double layer on the surface covered with a hydrophobic film. Equivalent circuit $C$ can describe impedance spectra in which up to three time constants appear.

For the same conditions as in Table 1, the use of the equivalent circuit $C$ gave the results presented in Table 2. In the equivalent circuit $\mathrm{C}$ there are no elements $R_{\mathrm{f}}$ and $C_{\mathrm{f}}$, there is no 
need to divide all processes into processes in pores and on a coated surface. For example, the capacitance $C_{\mathrm{dl}}$ is the double-layer capacitance averaged over the entire surface of the electrode, determined by relation (4). As can be seen from the Table 2, the capacitance $C_{\mathrm{dl}}$ has values typical for solid electrodes.
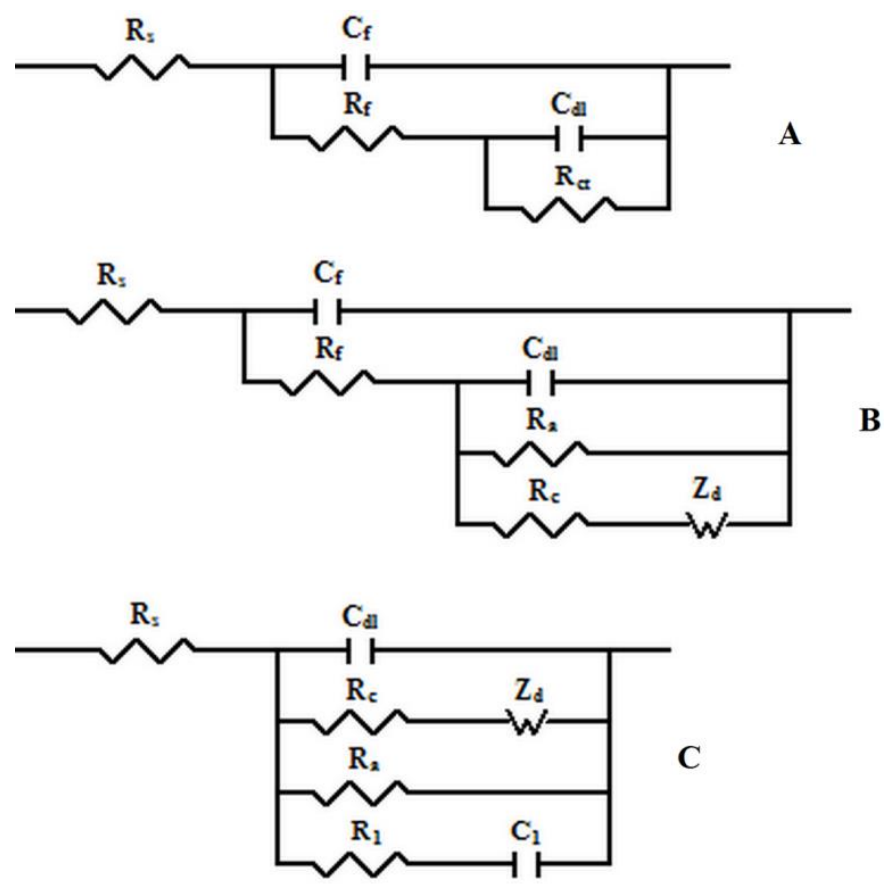

Figure 8. Analyzed equivalent electrical circuits.

Table 2. Values of the parameters of the equivalent circuit $C$ for a steel electrode with SHPC in NACE solution.

\begin{tabular}{cccccccc}
\hline \multirow{2}{*}{ Parameter } & \multicolumn{7}{c}{ Exposure time, $\mathbf{h}$} \\
\cline { 2 - 7 } & 0.25 & 24 & 48 & 72 & 96 & 120 & 168 \\
\hline$C_{\mathrm{dl}}, \mu \mathrm{F} / \mathrm{cm}^{2}$ & 10 & 49 & 72 & 31 & 61 & 48 & 54.4 \\
$R_{\mathrm{c}}, \mathrm{Ohm} \cdot \mathrm{cm}^{2}$ & 43 & 4.3 & 2.8 & 2.5 & 2.0 & 2.1 & 2.1 \\
$R_{\mathrm{d}}, \mathrm{Ohm} \cdot \mathrm{cm}^{2}$ & 1170 & 12630 & 11520 & 11700 & 14400 & 12860 & 13250 \\
$\tau_{\mathrm{d}}, \mathrm{s}$ & 23 & 118 & 136 & 152 & 206 & 178 & 189 \\
$p_{\mathrm{d}}$ & 0.40 & 0.67 & 0.70 & 0.73 & 0.73 & 0.74 & 0.75 \\
$R_{\mathrm{a}}, \mathrm{Ohm} \cdot \mathrm{cm}^{2}$ & - & 610 & 740 & 817 & 993 & 1130 & 1230 \\
$R_{1}, \mathrm{Ohm} \cdot \mathrm{cm}^{2}$ & 55 & 128 & 66 & 63 & 39 & 31.4 & 34.4 \\
$C_{1}, \mu \mathrm{F} / \mathrm{cm}^{2}$ & 13 & 166 & 230 & 320 & 412 & 513 & 614 \\
$s, \%$ & 3.0 & 0.27 & 0.21 & 0.41 & 0.29 & 0.28 & 0.56 \\
\hline
\end{tabular}


Equivalent circuits $\mathrm{B}$ and $\mathrm{C}$ contain the same number of elements, but circuit $\mathrm{C}$ is characterized by smaller deviations of the calculated impedance values from the experimental ones, although in some cases the root-mean-square deviations for circuits $\mathrm{B}$ and $\mathrm{C}$ are approximately the same. It should be emphasized that the high accuracy of approximating the experimental impedance spectra by circuits $\mathrm{B}$ and $\mathrm{C}$, in contrast to circuit A, is achieved without using constant phase elements.

Table 3. Values of the parameters of the equivalent circuit $\mathrm{C}$ for a steel electrode with SHPC in NACE + $400 \mathrm{mg} / \mathrm{L}$ solution.

\begin{tabular}{cccccccc}
\hline \multirow{2}{*}{ Parameter } & \multicolumn{7}{c}{ Exposure time, h } \\
\cline { 2 - 8 } & 0.25 & 24 & 48 & 72 & 96 & 120 & 168 \\
\hline$C_{\mathrm{dl}}, \mu \mathrm{F} / \mathrm{cm}^{2}$ & 750 & 510 & 408 & 314 & 597 & 840 & 613 \\
$R_{\mathrm{c}}, \mathrm{Ohm} \cdot \mathrm{cm}^{2}$ & 1.8 & 10 & 23.7 & 13.9 & 7.6 & 5.2 & 0.6 \\
$R_{\mathrm{d}}, \mathrm{Ohm} \cdot \mathrm{cm}^{2}$ & 1340 & 1720 & 2010 & 1180 & 797 & 520 & 620 \\
$\tau_{\mathrm{d}}, \mathrm{s}$ & 12 & 227 & 310 & 163 & 210 & 92 & 174 \\
$p_{\mathrm{d}}$ & 0.71 & 0.62 & 0.55 & 0.65 & 0.62 & 0.53 & 0.50 \\
$R_{\mathrm{a}}, \mathrm{Ohm} \cdot \mathrm{cm}^{2}$ & 740 & 956 & 1980 & 1060 & 3480 & 3500 & 3070 \\
$R_{1}, \mathrm{Ohm} \cdot \mathrm{cm}^{2}$ & 73 & 7.1 & 12.2 & 4.3 & 3.1 & 4.2 & 6.2 \\
$C_{1}, \mu \mathrm{F} / \mathrm{cm}^{2}$ & 208 & 768 & 485 & 1180 & 1620 & 1350 & 637 \\
$s, \%$ & 0.06 & 3.1 & 0.41 & 1.2 & 0.8 & 0.45 & 0.21 \\
\hline
\end{tabular}

Table 4. Values of the parameters of the equivalent circuit $\mathrm{C}$ for a steel electrode with SHPC in $\mathrm{NaCl}$ $(50 \mathrm{~g} / \mathrm{L})$ solution.

\begin{tabular}{cccccc}
\hline \multirow{2}{*}{ Parameter } & \multicolumn{5}{c}{ Exposure time, $\mathbf{h}$} \\
\cline { 2 - 5 } & 0.25 & 24 & 48 & 72 & 168 \\
\hline$C_{\mathrm{dl}}, \mu \mathrm{F} / \mathrm{cm}^{2}$ & 3.3 & 448 & 393 & 575 & 802 \\
$R_{\mathrm{c}}, \mathrm{Ohm} \cdot \mathrm{cm}^{2}$ & 15 & 1.1 & 1.2 & 0.8 & 0.5 \\
$R_{\mathrm{d}}, \mathrm{Ohm} \cdot \mathrm{cm}^{2}$ & 2770 & 2630 & 2560 & 2520 & 2230 \\
$\tau_{\mathrm{d}} \mathrm{s}$ & 6 & 139 & 158 & 166 & 133 \\
$p_{\mathrm{d}}$ & 0.59 & 0.69 & 0.66 & 0.64 & 0.72 \\
$R_{\mathrm{a}}, \mathrm{Ohm} \cdot \mathrm{cm}^{2}$ & 1950 & 1930 & 4000 & 3190 & 2790 \\
$R_{1}, \mathrm{Ohm} \cdot \mathrm{cm}^{2}$ & 16.4 & 89 & 97 & 115 & 196 \\
$C_{1}, \mu \mathrm{F} / \mathrm{cm}^{2}$ & 3.8 & 1910 & 2990 & 3490 & 3230 \\
$s, \%$ & 1.9 & 1.8 & 3.0 & 1.6 & 0.5 \\
\hline
\end{tabular}


Table 5. Values of the parameters of the equivalent circuit $\mathrm{C}$ for a steel electrode with SHPC in $\mathrm{NaCl}$ $(50 \mathrm{~g} / \mathrm{L})+400 \mathrm{mg} / \mathrm{L} \mathrm{H}_{2} \mathrm{~S}$ solution .

\begin{tabular}{cccccc}
\hline \multirow{2}{*}{ Parameter } & \multicolumn{5}{c}{ Exposure time, $\mathbf{h}$} \\
\cline { 2 - 6 } & 0.25 & 24 & 48 & 72 & 168 \\
\hline$C_{\mathrm{dl}}, \mu \mathrm{F} / \mathrm{cm}^{2}$ & 185 & 93 & 81 & 119 & 113 \\
$R_{\mathrm{c}}, \mathrm{Ohm} \cdot \mathrm{cm}^{2}$ & 6.7 & 0.7 & 0.8 & 0.4 & 0.4 \\
$R_{\mathrm{d}}, \mathrm{Ohm} \cdot \mathrm{cm}^{2}$ & 2410 & 12180 & 10960 & 7690 & 5620 \\
$\tau_{\mathrm{d}}, \mathrm{s}$ & 174 & 118 & 99 & 141 & 112 \\
$p_{\mathrm{d}}$ & 0.68 & 0.54 & 0.58 & 0.52 & 0.54 \\
$R_{\mathrm{a}}, \mathrm{Ohm} \cdot \mathrm{cm}^{2}$ & 738 & 7710 & 2240 & 2460 & 12480 \\
$R_{1}, \mathrm{Ohm} \cdot \mathrm{cm}^{2}$ & 2.7 & 9.6 & 10.9 & 13.9 & 10.2 \\
$C_{1}, \mu \mathrm{F} / \mathrm{cm}^{2}$ & 458 & 180 & 182 & 188 & 197 \\
$s, \%$ & 13.1 & 15.9 & 23 & 11.4 & 15.2 \\
\hline
\end{tabular}

In connection with some advantages of the equivalent circuit $\mathrm{C}$, further analysis is carried out using this model. For NACE $+\mathrm{H}_{2} \mathrm{~S}, \mathrm{NaCl}, \mathrm{NaCl}+\mathrm{H}_{2} \mathrm{~S}$ solutions, the values of the parameters of circuit $\mathrm{C}$ are shown in Tables $3-5$. Note that in a solution of $50 \mathrm{~g} / \mathrm{L} \mathrm{NaCl}$ $+400 \mathrm{mg} / \mathrm{L} \mathrm{H}_{2} \mathrm{~S}$, the accuracy of the approximation of the impedance spectra using the equivalent circuit $\mathrm{C}$ was not high enough (Table 5).

\section{Results and Discussion}

The impedance of an electrode corroding in an $\mathrm{H}_{2} \mathrm{~S}$-containing environment depends on many factors: the concentration of hydrogen sulfide in the solution, the composition and $\mathrm{pH}$ of the solution, the temperature, the exposure time of the samples, the composition and structure of the films of corrosion products (iron sulfides), the presence of oxide layers on the electrode, etc. [32]. To a large extent, this probably also applies to an electrode with a superhydrophobic coating, which is not perfect, contains defects, and for such a coating contact of the substrate with the medium is not completely excluded [33].

The use of equivalent circuit $\mathrm{C}$ for all cases $\left(\mathrm{NaCl}\right.$ solution without $\mathrm{H}_{2} \mathrm{~S}$ and with added $\mathrm{H}_{2} \mathrm{~S}$, NACE solution without $\mathrm{H}_{2} \mathrm{~S}$ and with added $\mathrm{H}_{2} \mathrm{~S}$ ) assumes that the nature of the cathodic and anodic processes in these media is the same. The cathodic process in sodium chloride solution is oxygen reduction. The NACE solution is more acidic ( $\mathrm{pH} 3.6)$, there is a thermodynamic possibility of the hydrogen evolution reaction (HER) at $E_{\text {cor }}$. In addition, amorphous iron sulfides exhibit a significant catalytic effect with respect to HER (in particular, in an acetate buffer solution, $\mathrm{pH}$ 4.55) [34]. Therefore, there is reason to believe that in a NACE $+400 \mathrm{mg} / \mathrm{L} \mathrm{H}_{2} \mathrm{~S}$ solution, the reactions of hydrogen evolution and oxygen electroreduction can proceed in parallel. Due to the very low concentration of hydrogen ions, diffusion restrictions will be observed for the HER, as well as for the $\mathrm{O}_{2}$ reduction reaction. 
Therefore, the cathodic reaction of $\mathrm{H}^{+}$discharge in NACE solution and $\mathrm{O}_{2}$ reduction in $\mathrm{NaCl}$ and NACE solutions can be modeled by the same Ershler-Randles circuit, and measurements in NACE solution will give only effective parameters of diffusion impedance. The assumption that the anodic reaction mechanism remains unchanged when hydrogen sulfide is added to a $\mathrm{NaCl}$ or NACE solution is not entirely justified. At the same time, there is evidence that the same equivalent circuit can be used in solutions without $\mathrm{H}_{2} \mathrm{~S}$ and with the addition of $\mathrm{H}_{2} \mathrm{~S}$ [35].

The equivalent circuit $\mathrm{C}$, apparently, is characterized by three time constants: $R_{\mathrm{ct}} C_{\mathrm{dl}}$, $R_{1} C_{1}$, and diffusion time constant $\tau_{\mathrm{d}}=\delta^{2} / D$ ( $\delta$ is the thickness of the diffusion layer, $D$ is the diffusion coefficient). The charge transfer resistance $R_{\mathrm{ct}}$ in this case is $\left(1 / R_{\mathrm{c}}+1 / R_{\mathrm{a}}+1 / R_{1}\right)^{-1}$. Depending on the composition of the electrolyte, the exposure time, such ratios of the kinetic parameters of partial reactions can be created at which impedance spectra are observed with the number of time constants less than three.

The values of the parameters of the equivalent circuit obtained from the reduced impedance spectra at a short exposure time $(t=15 \mathrm{~min})$ differ significantly from the values at $t \geq 24 \mathrm{~h}$. In the NACE solution at $t=15 \mathrm{~min}$, the values of $C_{\mathrm{dl}}, R_{\mathrm{d}}, \tau_{\mathrm{d}}, p_{\mathrm{d}}$ are much less, and $R_{\mathrm{c}}$ is greater than at $t \geq 24 \mathrm{~h}$ (Table 2). In this case, the resistance $R_{\mathrm{a}}$ exceeds $10^{5} \mathrm{Ohm} \cdot \mathrm{cm}^{2}$ and cannot be reliably determined. Such a high value of $R_{\mathrm{a}}$ can be due to the fact that at low $\mathrm{t}$ the electrode is still covered with an oxide film. The same reason, as well as the manifestation of the superhydrophobic properties of the coating in full at a short contact time of the coating with the electrolyte, can explain the increased value of $R_{\mathrm{c}}$. At all $t$, the ratio $R_{\mathrm{c}}<<R_{\mathrm{d}}$ is fulfilled, that is, the cathodic process proceeds mainly with diffusion control.

In a NACE solution at $t \geq 24 \mathrm{~h}$, the resistance $R_{\mathrm{c}}$ decreases, and the resistance $R_{\mathrm{a}}$ increases with time (Table 2). These changes can be partly associated with a decrease in the corrosion potential with increasing $t$. The diffusion resistance $R_{\mathrm{d}}$ is almost constant in the $t$ interval from 24 to $168 \mathrm{~h}$ and has a rather high value (about $12 \mathrm{kOhm} \cdot \mathrm{cm}^{2}$ ). For other parameters of the diffusion impedance $\left(\tau_{\mathrm{d}}, p_{\mathrm{d}}\right)$, there is a tendency to increase with time. The parameter $p_{\mathrm{d}}$ differs markedly from 0.5 , which probably reflects the inhomogeneity of the diffusion layer. As the exposure time increases, the $R_{1}$ parameter decreases, and the $C_{1}$ parameter increases, and the time constant $\tau_{1}=R_{1} C_{1}$, which characterizes the relaxation rate of the surface coverage with the intermediate of the anodic process, is approximately constant in the interval of $t=24-168 \mathrm{~h}$ and is $15-20 \mathrm{~ms}$.

The corrosion rate $i_{\text {cor }}$ from the impedance data, as in the linear polarization method, is determined by relation (1). In the impedance spectroscopy method, the polarization resistance $R_{\mathrm{p}}$ in Eq. (1) is the limit to which the real component of the impedance tends when the frequency of the alternating current tends to zero. For equivalent circuit $\mathrm{C}$

$$
R_{\mathrm{p}}=\frac{R_{\mathrm{a}}\left(R_{\mathrm{c}}+R_{\mathrm{d}}\right)}{R_{\mathrm{a}}+R_{\mathrm{c}}+R_{\mathrm{d}}}
$$

Using the values of the Tafel slope coefficients obtained from polarization measurements in various media $[7,16,33]$ the $i_{\text {cor }}$ values were calculated at different 
exposure times according to Equation (1) using (5). The found corrosion rate of the steel electrode with SHPC in the NACE solution, in agreement with the increase in the diameter of the semicircles on the impedance plots (Figure 1), decreases with time and amounts to $19.5 \mu \mathrm{A} / \mathrm{cm}^{2}$ at $t=24 \mathrm{~h}$ and $10.5 \mu \mathrm{A} / \mathrm{cm}^{2}$ at $t=168 \mathrm{~h}$.

Addition of $400 \mathrm{mg} / \mathrm{L} \mathrm{H}_{2} \mathrm{~S}$ to the NACE solution causes significant changes in the impedance characteristics of the SHPC electrode. Compared to a solution without $\mathrm{H}_{2} \mathrm{~S}$, the values of $C_{\mathrm{dl}}, C_{1}$ are higher, the values of $R_{1}, R_{\mathrm{d}}, p_{\mathrm{d}}$ are lower. The resistance $R_{\mathrm{a}}$ at $t=15 \mathrm{~min}$ is relatively low, but with an increase in the exposure time it increases and at $t=168 \mathrm{~h}$ it is noticeably higher than in NACE. At the same time, a significant decrease in $R_{\mathrm{d}}$ is observed in the $t$ interval from 24 to $168 \mathrm{~h}$. The parameter $p_{\mathrm{d}}$ decreases with time to a value of 0.5 , which corresponds to diffusion to a homogeneous surface.It is possible that a long-term exposure of the electrode to $\mathrm{NACE}+\mathrm{H}_{2} \mathrm{~S}$ promotes formation of a dense film of conducting iron sulfides [36], and the reduction of oxygen (and hydrogen ions) occurs on the surface of this film. In other cases, when no dense conductive film is formed, diffusion of molecular oxygen occurs in an inhomogeneous medium (for example, through a porous layer of corrosion products), and the parameter $p_{\mathrm{d}}$ deviates from 0.5 . The time constant $\tau_{1}$ in a hydrogen sulfide-containing environment is $4-6 \mathrm{~ms}$, which is significantly less than in NACE without $\mathrm{H}_{2} \mathrm{~S}$. The corrosion rate calculated by (1), (5) increases with time and at $t=24 \mathrm{~h}$ is $11.7 \mu \mathrm{A} / \mathrm{cm}^{2}$, and at $t=168 \mathrm{~h}-24.5 \mu \mathrm{A} / \mathrm{cm}^{2}$.

In a $\mathrm{NaCl}$ solution, as in other media, the values of the parameters of the equivalent circuit at $t=15 \mathrm{~min}$ differ significantly from the values at long exposure times: the parameters $C_{\mathrm{dl}}, C_{1}, \tau_{\mathrm{d}}, p_{\mathrm{d}}$ are less, and $R_{\mathrm{c}}$ is greater than at $t \geq 24 \mathrm{~h}$. The diffusion impedance parameters change rather weakly at $t \geq 24 \mathrm{~h}$. The time constant $\tau_{1}$ at $t=24-168 \mathrm{~h}$ is 170 $630 \mathrm{~ms}$; this is the highest value among the 4 studied systems and it shows that the relaxation of the surface coverage with the intermediate of the anodic reaction in a sodium chloride solution is the slowest. The corrosion rate calculated according to (1), (5) decreases with time and at $t=24 \mathrm{~h}$ is equal to $10 \mu \mathrm{A} / \mathrm{cm}^{2}$, and at $t=72 \mathrm{~h}-4.1 \mu \mathrm{A} / \mathrm{cm}^{2}$.

In a solution of $\mathrm{NaCl}+400 \mathrm{mg} / \mathrm{L} \mathrm{H}_{2} \mathrm{~S}$, as already noted, the equivalent circuit $\mathrm{C}$ does not provide a high accuracy in describing the experimental impedance spectra $(s>10 \%$, Table 5), therefore, conclusions from the time dependences of the impedance parameters will be only approximate. Most of the parameters of circuit $\mathrm{C}$ at $t \geq 24 \mathrm{~h}$ vary weakly with time. As a result of the addition of $400 \mathrm{mg} / \mathrm{L} \mathrm{H}_{2} \mathrm{~S}$ to the $\mathrm{NaCl}$ solution, the relaxation time $\tau_{1}$ sharply decreases to 2-3 ms, that is, to values close to $\tau_{1}$ in the NACE $+\mathrm{H}_{2} \mathrm{~S}$ solution. Thus, in both solutions ( $\mathrm{NaCl}$ and NACE), the addition of hydrogen sulfide leads to a significant decrease in $\tau_{1}$. This, apparently, indicates that the nature of intermediate species in the anodic reaction on carbon steel in a solution without $\mathrm{H}_{2} \mathrm{~S}$ and in the presence of $\mathrm{H}_{2} \mathrm{~S}$ is different. At the same time, the number of steps in the anodic reaction can remain unchanged, which determines the applicability of the same equivalent circuit in all media.

Comparison of the $i_{\text {cor }}$ values at different exposure times with the time dependences of the equivalent circuit parameters allows us to make the assumption that in NACE and $\mathrm{NaCl}$ solutions without hydrogen sulfide, the factor determining the corrosion rate is the resistance 
of the anodic reaction $R_{\mathrm{a}}$, and in the NACE solution with the addition of $_{2} \mathrm{H}_{2} \mathrm{~S}$ it is the resistance $R_{\mathrm{d}}$ that characterizes the kinetics of the cathodic reaction.

\section{Conclusion}

The impedance spectra (frequency range of $10000-0.01 \mathrm{~Hz}$ ) of St3 steel electrodes with a superhydrophobic coating were obtained in solutions of $\mathrm{NaCl}(50 \mathrm{~g} / \mathrm{L})$ and NACE $(0.25 \mathrm{~g} / \mathrm{L}$ $\left.\mathrm{CH}_{3} \mathrm{COOH}, 5 \mathrm{~g} / \mathrm{L} \mathrm{NaCl}, \mathrm{pH}=3.6\right)$ in the absence and presence of $400 \mathrm{mg} / \mathrm{L} \mathrm{H}_{2} \mathrm{~S}$ at a corrosion potential and analyzed. Three equivalent circuits are considered, referring to two types of circuits. One type of equivalent circuit (Figure 7,a) assumes that the organic hydrophobic coating completely excludes a significant part of the surface from participating in electrochemical processes, and the impedance response is divided into the dielectric response of the coating in the form of the coating capacitance and the impedance of electrode reactions at the bottom of the pores in the coating. Another type of equivalent circuits (Figure 7,b) is a conventional parallel connection of the double-layer and Faraday impedances averaged over the entire surface. It is shown that the best agreement with experimental data is achieved for an equivalent circuit of the second type - circuit $\mathrm{C}$ (Figure 8), proposed in [31].

An explanation is given for the anomalous impedance spectra at a short exposure time of the electrode in the solution $(t=15 \mathrm{~min})$, when at low frequencies a significant decrease in impedance is observed compared to the impedance at medium frequencies. The parameters of the equivalent circuit $\mathrm{C}$ are calculated depending on the exposure time $t$ of the electrode in a corrosive environment $(t=0.25-168 \mathrm{~h})$. The observed changes in the parameters of the equivalent circuit over time can be associated mainly with the influence of two processes - gradual degradation of the hydrophobic coating and the growth of a film of corrosion products.

\section{Acknowledgements}

The authors are sincerely grateful to the staff of the Surface Forces Laboratory of the A.N. Frumkin Institute of Physical Chemistry and Electrochemistry of RAS, Academician of RAS L.B. Boinovich and Head of the laboratory Professor A.M. Emel'yanenko for assistance in applying the superhydrophobic agent onto the steel surface.

The experimental results were obtained using the equipment of the Center for Collective Use of Scientific Equipment of TSU named after G.R. Derzhavin.

\section{References}

1. Y. Wang, W. Wang, L. Zhong, J. Wang, Q. Jiang and X. Guo, Super-hydrophobic surface on pure magnesium substrate by wet chemical method, Appl. Surf. Sci., 2010, 256, 3837-3840. doi: 10.1016/j.apsusc.2010.01.037 
2. Z. Kang, Q. Ye, J. Sang and Y. Li, Fabrication of super-hydrophobic surface on copper surface by polymer plating, J. Mater. Process. Technol., 2009, 209, 4543-4547. doi: 10.1016/i.jmatprotec.2008.10.031

3. L.B. Boinovich, K.A. Emelyanenko, A.G. Domantovsky and A.M. Emelyanenko, Laser tailoring the surface chemistry and morphology for wear, scale and corrosion resistant superhydrophobic coatings, Langmuir, 2018, 34, no. 24, 7059-7066. doi: 10.1021/acs.langmuir.8b01317

4. B. He, N.A. Patankar and J. Lee, Multiple equilibrium droplet shapes and design criterion for rough hydrophobic surfaces, Langmuir, 2003, 19, no. 12, 4999-5003. doi: $10.1021 / \mathrm{la} 0268348$

5. D.A. Alpysbaeva, D.B. Vershok, A.M. Emel'yanenko, O.V. Batishchev, Yu.I. Kuznetsov and L.B. Boinovich, Superhydrophobization of low-carbon steel by surface coatings, Korroz.: Mater., Zashch. (Corrosion: materials, protection), 2013, no. 8, 42 (in Russian).

6. J. Ou, M. Liu, W. Li, M. Xue and C. Li, Corrosion behavior of superhydrophobic surfaces of Ti alloys in NaCl solutions, Appl. Surf. Sci., 2012, 258, 4724-4728. doi: 10.1016/j.apsusc.2012.01.066

7. V.I. Vigdorovich, L.E. Tsygankova, A.M. Emel'yanenko, M.N. Uryadnikova and E.Yu. Shel, The effect of superhydrophobic coating on the electrochemical behavior of carbon steel in chloride and hydrogen sulfide-chloride environments, Int. J. Corros. Scale Inhib., 2020, 9, no. 1, 171-181. doi: 10.17675/2305-6894-2020-10-1-10

8. V.I. Vigdorovich, L.E. Tsygankova, M.N. Uryadnikova, N.V. Shel and N. Alshikha, Protection of carbon steel by superhydrophobic coating in modal stratum water containing hydrogen sulfide, Vse Mater. Entsiklopedicheskii spravochnik (All materials. Encyclopedic Reference Book), 2020, no. 7, 41-47, (in Russian). doi: 10.31044/19946260-2020-0-7-41-47

9. L. Boinovich and A. Emel'yanenko, A wetting experiment as a tool to study the physicochemical processes accompanying the contract of hydrophobic and superhydrophobic materials with aqueous media, Adv. Colloid Interface Sci., 2012, 179_ 182, no. 1, 133-141. doi: $10.1016 /$ j.cis. 2012.06 .010

10. P. Agarwal, O.C. Moghissi, M.E. Orazem and L.H. Garcia-Rubio, Application of measurement models for analysis of impedance spectra, Corrosion, 1993, 49, no. 4, 278-289. doi: $\underline{10.5006 / 1.3316050}$

11. D.D. Macdonald, E. Sikora and G. Engelhardt, Characterizing electrochemical systems in the frequency domain, Electrochim. Acta, 1998, 43, no.1-2, 87-107. doi: 10.1016/S0013-4686(97)00238-7

12. P. Agarwal, M.E. Orazem and L.H. Garcia-Rubio, Application of measurement models to impedance spectroscopy. I. Demonstration of applicability, J. Electrochem. Soc., 1992, 139, no. 7, 1917-1927. doi: $10.1149 / 1.2069522$ 
13. G.J. Brug, A.L.G. van den Eeden, M. Sluyters-Rehbach and J.H. Sluyters, The analysis of electrode impedances complicated by the presence of a constant phase element, $J$. Electroanal. Chem. Interfacial Electrochem., 1984, 176, no. 1-2, 275-295. doi: 10.1016/S0022-0728(84)80324-1

14. M.E. Orazem and B. Tribollet, Electrochemical Impedance Spectroscopy, John Wiley \& Sons, Inc., 2008.

15. R. Kashkovskiy, K. Strelnikova and A. Fedotova, Application of electrochemical impedance spectroscopy to study hydrogen sulphide corrosion of steel and its inhibition: a review, Corros. Eng., Sci. Technol., 2019, 54, no. 6, 493-515. doi: $\underline{10.1080 / 1478422 X .2019 .1619979}$

16. V.I. Vigdorovich, L.E. Tsygankova, A.A. Uryadnikov, N.V. Shel, L.G. Knyazeva and E.D. Tanygina, The effect of nanocomposite superhydrophobic coating on corrosion and kinetics of electrode processes on steel in $0.5 \mathrm{M} \mathrm{NaCl}$ solution, Prot. Met. Phys. Chem. Surf., 2017, 53, no. 7, 1259-1264. doi: 10.1134/S2070205117070176

17. H.Y. Ma, C. Yang, S.H. Chen, Y.L. Jiao, S.X. Huang, D.G. Li and J.L. Luo, Electrochemical investigation of dynamic interfacial processes at 1-octadecanethiolmodified copper electrodes in halide-containing solutions, Electrochim. Acta, 2003, 48, 4277-4289. doi: 10.1016/j.electacta.2003.08.003

18. G. Li, H. Ma, Y. Jiao and S. Chen, An impedance investigation of corrosion protection of copper by self-assembled monolayers of alkanethiols in aqueous solution, J. Serb. Chem. Soc., 2004, 69, 791-805. doi: 10.2298/JSC0410791L

19. Y. Feng, S. Chen, J. You and W. Guo, Investigation of alkylamine self-assembled films on iron electrodes by SEM, FT-IR, EIS and molecular simulations, Electrochim. Acta, 2007, 53, 1743-1753. doi: 10.1016/j.electacta.2007.08.035

20. F. Cecchet, M. Marcaccio, M. Margotti, F. Paolucci, S. Rapino and P. Rudolf, Redox mediation at 11-mercaptoundecanoic acid self-assembled monolayers on gold, J. Phys. Chem. B, 2006, 110, 2241-2248. doi: 10.1021/jp054290n

21. A. Muthurasu and V. Ganes, Electrochemical characterization of Self-assembled Monolayers (SAMs) of silanes on indium tin oxide (ITO) electrodes - Tuning electron transfer behavior across electrode-electrolyte interface, J. Colloid Interface Sci., 2012, 374, 241-249. doi: 10.1016/j.jcis.2012.02.007

22. L.E. Tsygankova, V.I. Vigdorovich, Ya.R. Kim, V.I. Kichigin and A.V. Boldyrev, Inhibition of corrosion and hydrogenation of carbon steel by a number of inhibitors in weakly acidic media containing $\mathrm{H}_{2} \mathrm{~S}$ and $\mathrm{CO}_{2}$, Zh. Prikl. Khim. (J. Appl. Chem.), 2005, 78, no. 12, 1993-2001 (in Russian).

23. L.B. Boinovich, S.V. Gnedenkov, D.A. Alpysbaeva, V.S. Egorkin, A.M. Emel'yanenko, S.L. Sinebryukhov and A.K. Zaretskaya, Corrosion resistance of composite coatings on low-carbon steel containing hydrophobic and superhydrophobic layers in combination with oxide sublayers, Corros. Sci., 2012, 55, 238-245. doi: 10.1016/j.corsci.2011.10.023 
24. D. Yu, J. Tian, J. Dai and X. Wang, Corrosion resistance of three-layer superhydrophobic composite coating on carbon steel in sea water, Electrochim. Acta, 2013, 97, 409-419. doi: $10.1016 /$ j.electacta.2013.03.071

25. A.B. Radwan, A.M.A. Mohamed, A.M. Abdullah and M.A. Al-Maadeed, Corrosion protection of electrospun PVDF-ZnO superhydrophobic coating, Surf. Coat. Technol., 2016, 289, 136-143. doi: 10.1016/j.surfcoat.2015.12.087

26. X. Cui, G. Zhu, Y. Pan, Q. Shao, C.(x.) Zhao, M. Dong, Y. Zhang and Z. Guo, Polydimethylsiloxane-titania nano composite coating: fabrication and corrosion resistance, Polymer, 2018, 138, 203-210. doi: 10.1016/j.polymer.2018.01.063

27. T. Xiang, Y. Han, Z. Guo, R. Wang, S. Zheng, S. Li, C. Li and X. Dai, Fabrication of inherent anticorrosion superhydrophobic surfaces on metals, ACS Sustainable Chem. Eng., 2018, 6, 5598-5606. doi: 10.1021/acssuschemeng.8b00639

28. A. Bonnel, F. Dabosi, C. Deslouis, M. Duprat, M. Keddam and B. Tribollet, Corrosion study of a carbon steel in neutral chloride solutions by impedance techniques, $J$. Electrochem. Soc., 1983, 130, no. 4, 753-761. doi: 10.1149/1.2119798

29. A. Lasia, Electrochemical Impedance Spectroscopy and its Applications, Springer Science + Business Media, New York, 2014.

30. D.A. Harrington, The rate-determining step in electrochemical impedance spectroscopy, J. Electroanal. Chem., 2015, 737, 30-36. doi: 10.1016/j.jelechem.2014.06.003

31. Ya.R. Kim, L.E. Tsygankova and V.I. Kichigin, Inhibition of corrosion and hydrogenation of steel in model stratum waters, Korroz.: Mater., Zashch. (Corrosion: materials, protection), 2005, no. 8, 30-37 (in Russian).

32. R.V. Kashkovskiy and Yu.I. Kuznetsov, Application of electrochemical impedance spectroscopy to study the structure and properties of sulfide films on steel. Part 1, Korroz.: Mater., Zashch. (Corrosion: materials, protection), 2012, no. 4, 38-44 (in Russian).

33. V.I. Vigdorovich, L.E. Tsygankova, M.N. Uryadnikova, K.A. Emel'yanenko, E.V. Chulkova and A.A. Uryadnikov, The influence of the medium on the protective efficiency of superhydrophobic nano composite coatings on carbon steel, Korroz.: Mater., Zashch. (Corrosion: materials, protection), 2020, no. 10, 42-48 (in Russian). doi: 10.31044/1813-7016-2020-0-10-42-48

34. T.Våland, T. Burchardt and S.F. van der Meer, The hydrogen evolution and corrosion of amorphous $\mathrm{FeS}_{\mathrm{x}}$ films, Corros. Sci., 2001, 43, 147-156. doi: 10.1016/S0010938X(00)00059-7

35. E. Abelev, T.A. Ramanarayanan and S.L. Bernasek, Iron corrosion in $\mathrm{CO}_{2} /$ brine at low $\mathrm{H}_{2} \mathrm{~S}$ concentrations: an electrochemical and surface science study, J. Electrochem. Soc., 2009, 156, C331-C339. doi: 10.1149/1.3160373

36. C.M. Menendez, V. Jovancicevic, S. Ramachandran, M. Morton and D. Stegmann, Assessment of corrosion under iron sulfide deposits and $\mathrm{CO}_{2} / \mathrm{H}_{2} \mathrm{~S}$ conditions, Corrosion, 2013, 69, no. 2, 145-156. doi: $10.5006 / 0441$ 